\title{
GABOR WAVELETS AND MORPHOLOGICAL SHARED WEIGHTEd NeURAL NeTWORK BASED AUTOMATIC FACE RECOGNITION
}

\author{
Chandrappa $\mathrm{D} \mathrm{N}^{1}$ and Ravishankar $\mathrm{M}^{2}$ \\ ${ }^{1}$ Department of Electronics \& Communication Engineering, SJB Institute of Technology, \\ Bangalore, India \\ chandrappa.dnegmail.com \\ ${ }^{2}$ Department of Information Science \& Engineering, DSCE, Bangalore, India \\ ravishankarmen@gmail.com
}

\begin{abstract}
Automatic face recognition system is an important component of intelligent human computer interaction systems for biometric. It is a attractive biometric approach, to distinguish one person from another. To perform Automatic face recognition system, the hybrid approach called Gabor Wavelets face detection and Morphological Shared Weighted Neural Network based Face Recognition. Face detection is performed by using Gabor filter feature extraction. The feature vector based on Gabor filters is used to reduce feature subspace. The detected face regions are given as input to Morphological Shared-weight Neural Network $(M S N N)$ which performs face recognition. Being non linear and translation invariant, the MSNN can create better generalization during face recognition. Feature extraction for MSNN is performed on hit-miss transforms that are independent of gray-level shifts. Then the output is learned by interacting with the classification process. The system is experimented on standard datasets and also on our own dataset of image owing to different illumination conditions and cluttered background in non frontal images with a crowded scene with different conditions. Face detection is performed on a cluttered background and crowded scene where a false negative and false positive is detected. The MSNN recognize ignores all false positives and false negatives from face detection and performs human faces in unconstrained environments, and multi-view recognition.
\end{abstract}

\section{KEYWORDS}

Face detection, Face recognition, Feed Forward Neural Network (FFNN), Gabor Wavelets, MSNN

\section{INTRODUCTION}

Digital Image Processing [1] is an ever expanding and dynamic area with applications reaching out into our everyday life such as, surveillance, biometrics and many more areas. Biometrics are the emerging field of Bioengineering; it is the automated method of recognizing a person based on a physiological or behavioural characteristic. Automatic recognition of people has received increasing attention from the Computer Vision, Machine Learning, Pattern Recognition and Computer Graphics communities during the last few decades of Survey [3]. Automatic Recognition of people is a challenging problem [4], Which is various real life applications in different fields such as, law enforcement, security systems and availability of powerful and lowcost desktop and embedded computing systems has created an enormous interest in automatic processing of digital images. 
Signal \& Image Processing : An International Journal (SIPIJ) Vol.4, No.4, August 2013

The rest of the paper is organized as follows, Section 2 describes about proposed method, Section 3 explains about Gabor Wavelets and Neural Networks for Face detection, section 4 explains about Morphological Shared Weighted Neural Network for Face Recognition, Section 5 demonstrates experimentation and results. Finally, conclusions are drawn in Section 6.

\section{Proposed MeThod}

The proposed Automatic Face Recognition technique comprises two main stages. The primary stage is Face Detection which performs face detection using Gabor Wavelets for feature extraction and Neural Networks for classification. The detected faces are cropped and given as input to the second stage for face recognition. In this stage Face Recognition with Feature extraction by MSNN and Feature matching is performed. The entire process of Automatic Face Recognition is illustrated in Fig. 1.

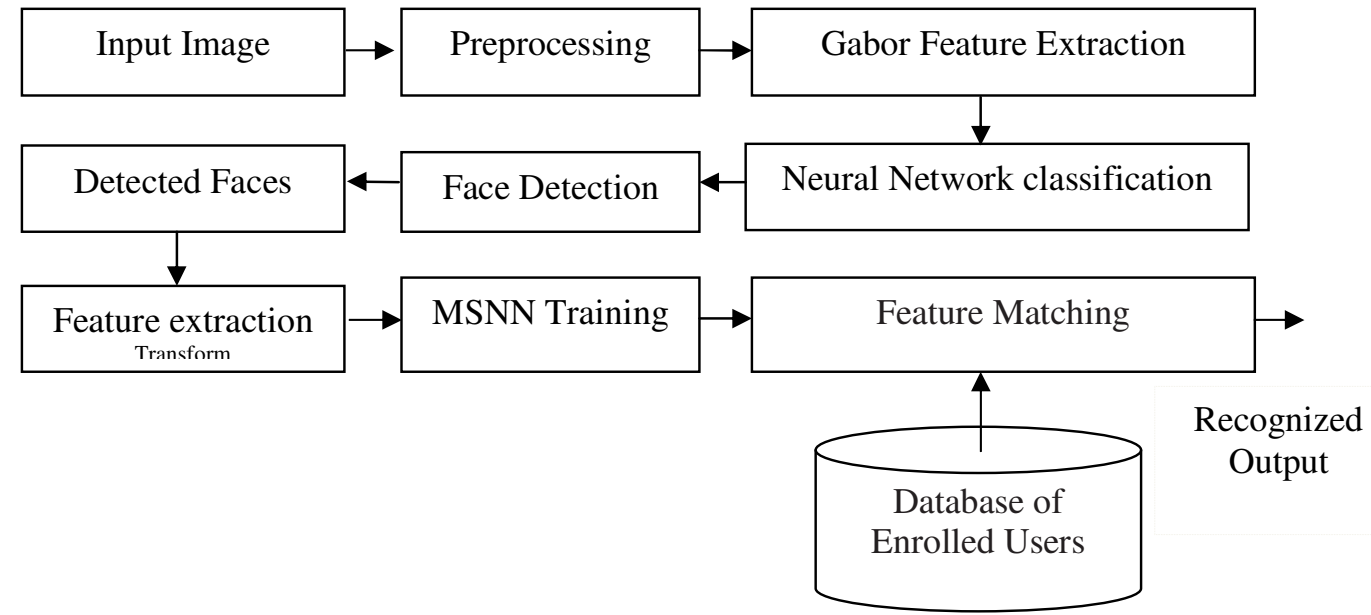

Figure 1. Block Diagram of the proposed Approach

\section{FACE DETECTION}

In this stage, Face detection is achieved by detecting areas that indicate a human face region. Face detection is based on using Gabor feature extraction and Neural Network classification to detect faces independently of the cluttered background and unconstraint environment with multi view face images.

\subsection{Gabor Wavelets}

The input Image is pre-processing, the histogram equalization for better contrast image Preprocessed image is convolved with Gabor filters by multiplying image by Gabor filters in frequency domain. Gabor filters are widely used in image analysis and computer vision. The Gabor filters transform provides an effective way to extract information in the form of space and frequency. The Gabor image representation is obtained by computing the convolution of the original image with several Gabor wavelets, which can be defined as follows

$$
\psi_{i}(\vec{x})=\frac{\left\|\vec{k}_{i}\right\|^{2}}{\sigma^{2}} e^{-\frac{\left\|\vec{k}_{\mathrm{i}}\right\|^{2}\left\|\vec{x}^{2}\right\|^{2}}{2 \sigma^{2}}}\left[e^{j \vec{k}_{\mathrm{i}} \vec{x}}-e^{-\frac{\sigma^{2}}{2}}\right]
$$


Signal \& Image Processing : An International Journal (SIPIJ) Vol.4, No.4, August 2013

Each $\Psi \mathrm{i}$ is a plane wave characterized by the vector ki enveloped by a Gaussian function, where $\sigma$ is the standard deviation of this Gaussian. The frequency of $i_{\text {th }}$ filter is given by the wave vector, having a scale and orientation given by $(\mathrm{k} v, \theta \mu)$, where $\mu$ and $v$ defines the orientation and scale of Gabor kernels. The first term in the brackets determines the oscillatory part of the kernel, and the second term compensates for the DC value of the kernel. Subtracting the DC response, Gabor filters becomes insensitive to the overall level of illumination.

$$
\begin{gathered}
\vec{k}_{i}=\left(\begin{array}{l}
k_{i x} \\
k_{i v}
\end{array}\right)=\left(\begin{array}{c}
k_{v} \cos \theta_{\mu} \\
k_{v} \sin \theta_{\mu}
\end{array}\right) \\
R_{i}(\vec{x})=\int I(\vec{x}) \Psi_{i}(\overrightarrow{\mathrm{x}}-\overrightarrow{\mathrm{x}}) \mathrm{dx}
\end{gathered}
$$

Where $\mathrm{I}\left({ }^{\vec{x}}\right)$ is the image intensity value at $\vec{x}, \mathrm{i}=1, \ldots 40$. Figure. 2 shows the real part of Gabor kernels, at 5 different spatial frequency $(v=0,1,2,3,4)$ and 8 orientations $(\mu=0,1,2,3,4,5,6$, 7) respectively.

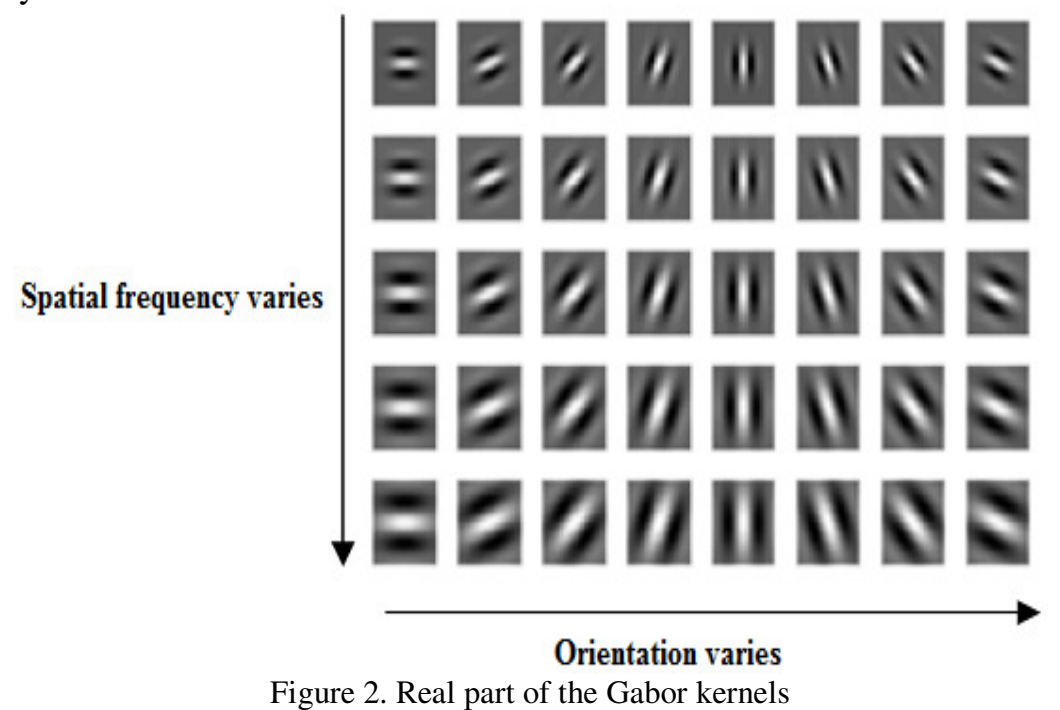

\subsection{Gabor Wavelet Representation of Faces}

The Gabor wavelet representation of an face image is the convolution of the image with a Gabor kernels. Gabor wavelet transforms allowing the description of both the spatial frequency structure and spatial relations. Convolving the image with complex Gabor filters with 5 scales and 8 orientations with both amplitude and phase. In Figure. 3, an input face image and the amplitude of the Gabor filter responses are shown below. 


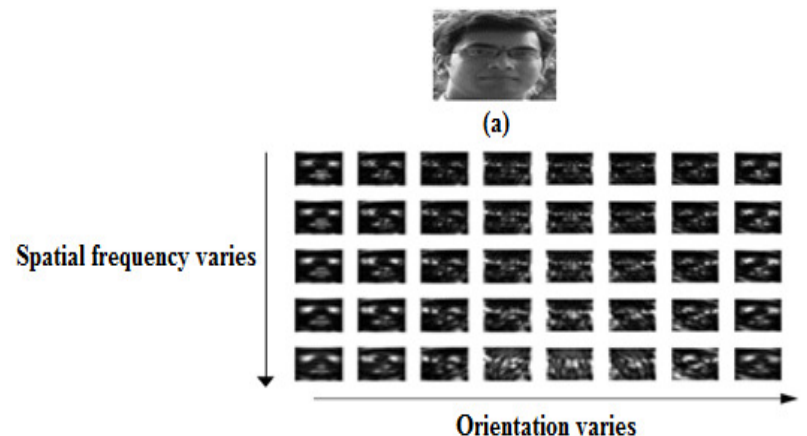

(b)

Figure. 3. Gabor filter responses of a face image a) Original face image (b) filter responses

The filter reaction outputs exhibits strong locality, scale and orientation selectivity, such characteristics produce salient local features such as the mouth, nose, eyebrow, jaw line, cheekbones as shown in Figure.

4 , that is suitable for visual event recognition, and hence making them a suitable choice for feature extraction of images.
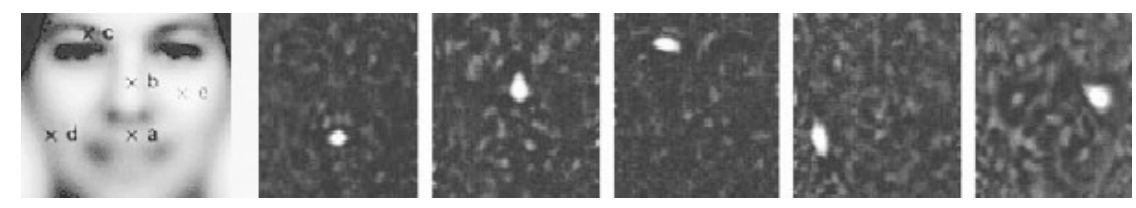

Figure 4. Local features of the face (a) mouth, (b) nose, (c) eyebrow, (d) jaw line,

\subsection{Gabor Feature Extraction}

(e) cheekbone

The Feature extraction stages of the proposed method have two main steps: (a) Feature point localization step (b) Feature vector generation step.

\section{(a) Feature point localization}

As the Gabor filter response have 40 different local, scale and orientation features, the dimensionality of the Gabor transformed image space is very high. For the responses of the face image to Gabor filters, peaks are found by searching the locations in a window $\mathrm{W}_{0}$ of size $\mathrm{W} \times \mathrm{W}$. A feature point is located at $\left(\mathrm{x}_{0}, \mathrm{y}_{0}\right)$, if

$$
\begin{gathered}
R_{j}\left(X_{0}, Y_{0}\right)=\max _{(x, y) \in W_{0}}\left(R_{j}\left(x_{i} y\right)\right) \\
R_{j}\left(X_{0}, Y_{0}\right)>\frac{1}{N_{2} N_{2}} \sum_{X=1}^{N_{1}} \sum_{Y=1}^{N_{2}} R_{j}(X, Y)
\end{gathered}
$$

$$
\text { Where } j=1,2 \ldots 40
$$

Where, $R_{j}$ is the response of the face image to the $j_{t h}$ Gabor filter. N1 N2 is the size of face image. Window size $\mathrm{W}$ is one of the important parameters of proposed algorithm, and it must be chosen small enough to capture the important features and large enough to avoid redundancy.

\section{(b) Feature vector generation}

Feature vectors are generated at the feature points as a composition of Gabor wavelet transform coefficients. $K_{\text {th }}$ feature vector of $i_{\text {th }}$ reference face is defined as, 


$$
V_{t, k}=\left\{X_{k}, Y_{k}, R_{u},\left(X_{k}, Y_{k}\right) j=1, \ldots \ldots, 40\right\}
$$

While there are 40 Gabor filters, feature vectors have 42 components as shown in Figure. 5. The first two components of feature vectors are very important during matching process. The remaining 40 components are the samples of the Gabor filter responses at that point. The Feature vectors, as the samples of Gabor wavelet transform at feature points, allow representing both the spatial frequency structure and spatial relations of the local image region around the corresponding feature point. Feature vectors are generated from windows of input face image, data will be transformed into the classification.

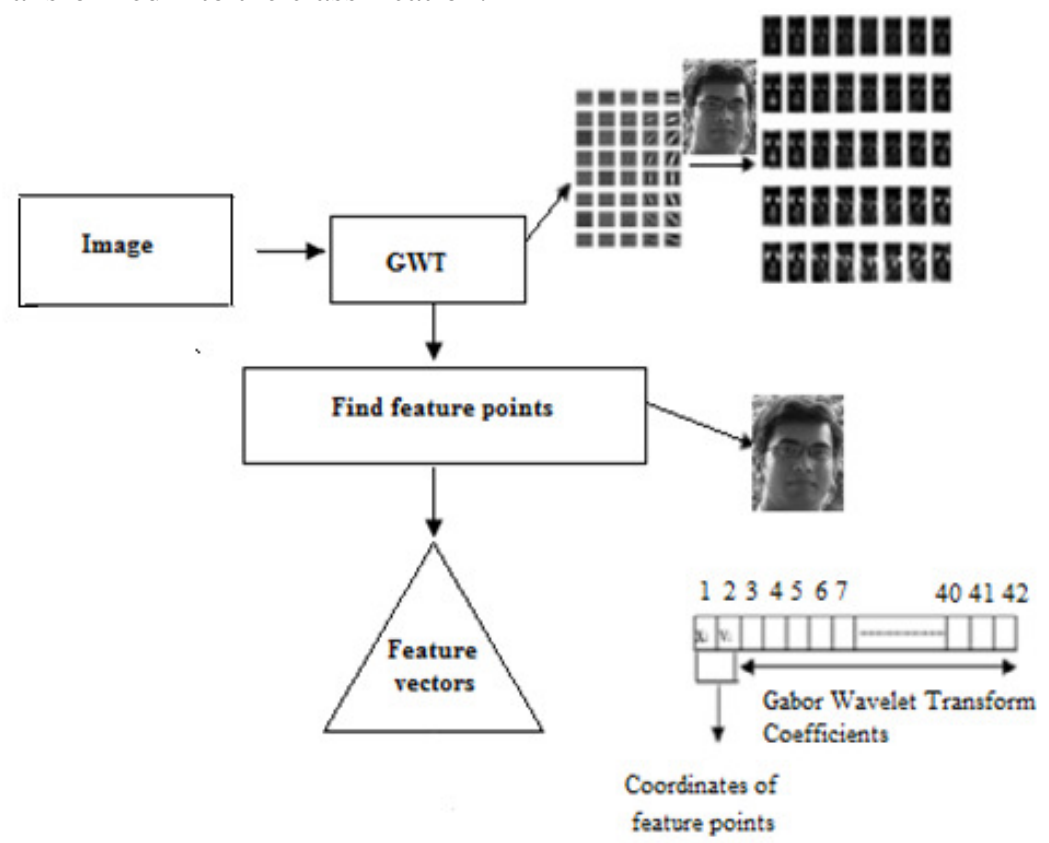

Figure. 5. Feature extraction of face detection

\subsection{Classification with Neural Networks}

The face detection was assured by a non linear classifier which is the neural networks. The advantage of using neural network for detection is the feasibility of training a system in very complex conditions. However, the network architecture has to be varied (number of layers and nodes) to get good performances. Feed forward net is used with the input vector constituting the dimension of the each image in the database is $27 \times 18$ pixels representing human faces and nonface.

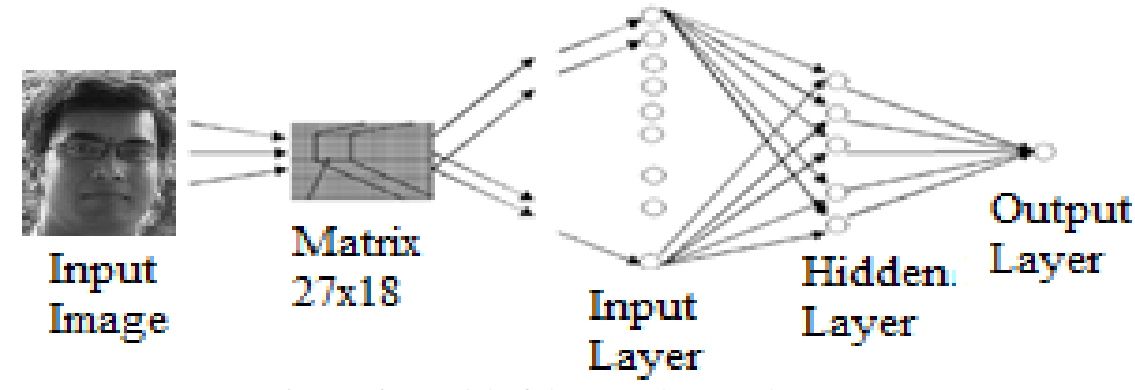

Figure 6. Model of the neural network System 
Face Features are a cell array contains the result of the convolution of the image with each of the forty Gabor filters. These matrices have concatenated to form a $135 \times 144$ matrix of complex numbers having 19,440 pixels. Only the magnitude of the result is required to remove corners as they contain no such information regarding faces. So $135 \times 144$ matrix is reduced to $45 \times 48$ matrix containing 2160 input layers. Feature vectors are generated from windows of input face image, data will be transformed into the form of a vector. The applied neural network is a two-layer Perceptron neural network with 100 neurons in the first layer and one neuron in the second or output layer. Then a matrix which every column of it is characteristic of one of training photos is used for network training accompanied by the desirable response of each column which is 0.9 for face vectors and -0.9 for non face vectors. Finally identify the face regions and rectangle box to mark the detected face as shown in Figure. 7. Once we get the face regions and crop them and save each as individual images for further processing for face recognition.

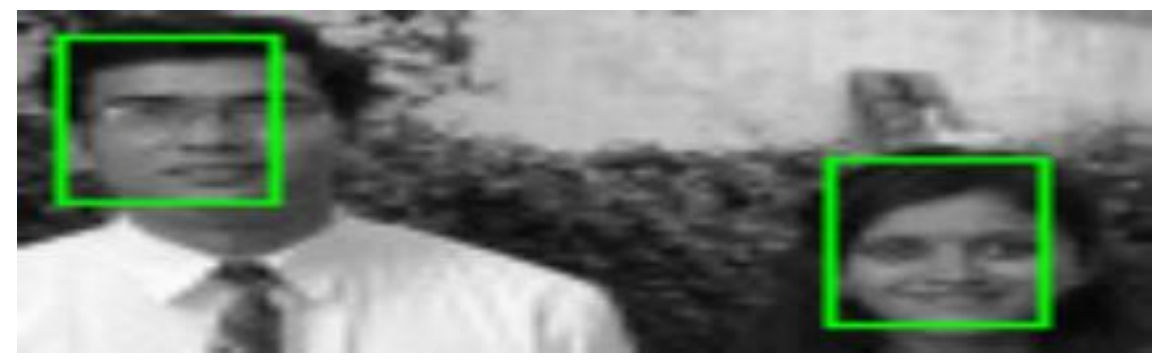

Figure 7. Final Detected faces

\section{FACE RECOGNITION}

Humans can recognize faces with little difficulty even in complex background environments. Therefore, the human face is a natural and attractive biometric. Most approaches to automate human face recognition typically analyze face images automatically. Experimental results can thus be collected and approaches are evaluated quickly by using MSNN method. The detected faces from detection algorithm are cropped and taken as input to the MSNN. MSNN model consists of two stage heterogeneous neural networks a feature extraction stage and classification stage. Designing an MSNN structure that is able to accomplish face recognition was extended from previous MSNN research in the area of Automatic Target Recognition (ATR) [10]. The MSNN is suited to object recognition, especially when presented with various, lighting levels, of the target image. Most other face recognition methods utilize feature extraction methods to determine a feature vector encoding representing a face; then standard matches are made to the database as determined by a similarity measure.

\subsection{Feature Extraction}

The complete feature extraction stage is one or more of these feature extraction layers, each feeding its output to the next layer as input. The first feature extraction layer uses the input subimage as input, and the last layer's output is the input for the classification stage. In each feature extraction layer a set of Hit and Miss structuring elements is shared amongst all feature maps. The feature map nodes perform the gray scale erosion and dilation known as the hit-miss transform. Each input face image is eroded by a hit structuring element and dilated by a miss structuring element separately (Figure. 8). The result from this process forms the feature map, which becomes the direct input to a back propagation network. 
Signal \& Image Processing : An International Journal (SIPIJ) Vol.4, No.4, August 2013

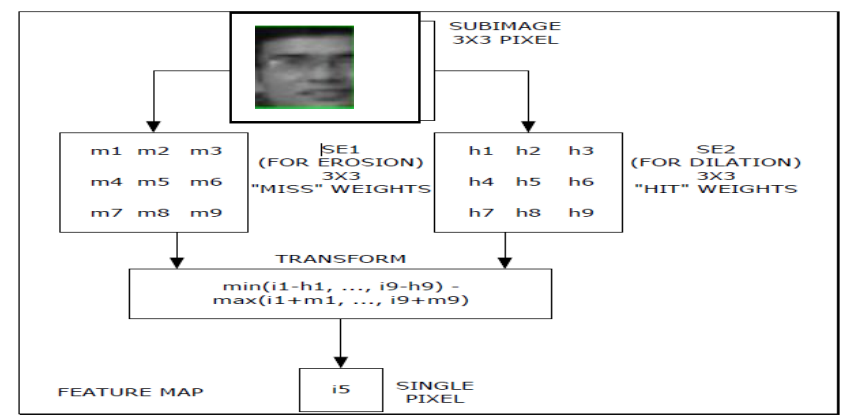

Figure. 8 Feature extraction layer

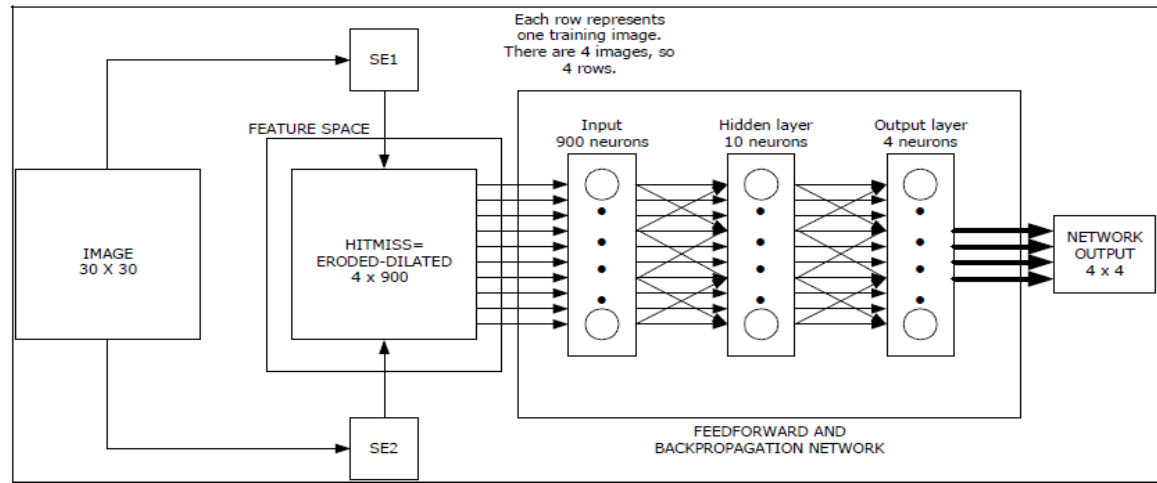

Figure. 9. Proposed MSNN architecture

The MSNN architecture is shown in Figure. 9. The feature map from the feature extraction stage is fed into the back propagation network. The net sums entering the neurons are increased by adding a bias. The output is subtracted by the target vector, and this error is used to calculate the correction term for the output layer. Since the network has one hidden layer, the output correction term is passed back to this hidden layer, which uses it to calculate its own correction term. Weights are then adjusted respectively at the output layer and the hidden layer. The final weights set is then multiplied and sigmoid transformed again with the original input to derive the final output for the entire training process. The feature is trained and compared with those of other face images to determine the closest match.

\subsection{Feature matching}

Face recognition is performed by comparing thresholds. All the images were trained a few times to record their output ranges. These were used to set recognition thresholds for each image. The output for every individual set is given by an array of four values, for instance $\left[\begin{array}{llll}0.7 & 0.8 & 0.1 & 0.2\end{array}\right]$. During face recognition, the classifier receives two sets of outputs, each belonging to the test set and the training set. This process starts by finding the maximum value among each output array (i.e. 0.8 for the example array given above). This maximum value for every image is then compared against all the thresholds. If a value falls within a particular threshold, an integer identifying the associated threshold is entered into the recognition array. This array, called "flag", is finally used to retrieve the corresponding face image from the identity dataset. 
Signal \& Image Processing : An International Journal (SIPIJ) Vol.4, No.4, August 2013

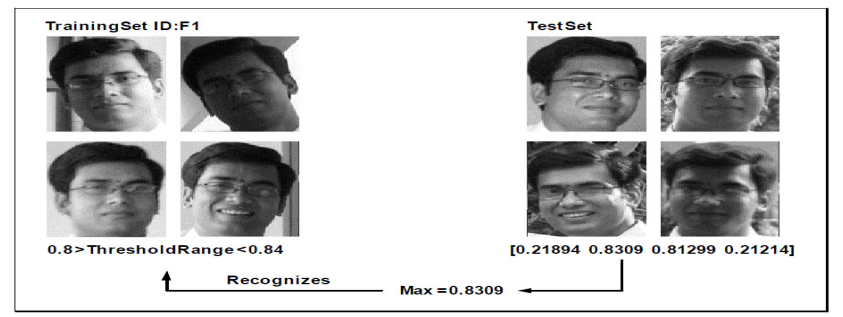

Figure. 10. Recognition by threshold matching.

\section{EXPERIMENTAL RESULTS AND DisCUSSION}

In this paper, the MATLAB simulated results are performed to verify the effectiveness of the proposed reorganization system. The system is experimented on standard CMU, USD datasets and also on our own dataset of image owing to different illumination conditions and cluttered background in non frontal images with crowded scene as shown in Fig. 11. Face detection performed on a crowded scene where a false negative and false positive is detected. False positive error includes those locations of face which has been detected as face wrongly. False negative error locations in the face in which there is face, however has not been recognized as face. The MSNN ignores all false positives and false negatives from face detection and performs face recognition only for valid face. Based on the Experimental output images, the face detection rate is high and the false acceptance rate is low. The Statistical data of face detection and recognition as shown in Table.1

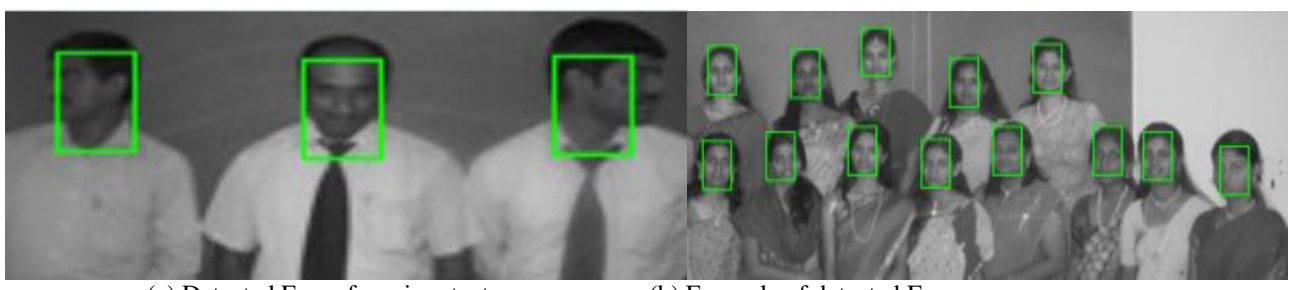

(a) Detected Faces from input stage

(b) Example of detected Faces

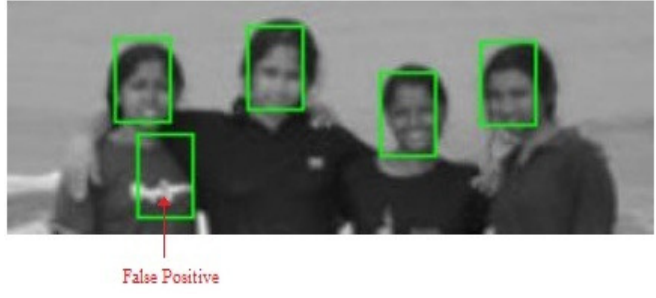

(c) Face detection with false positive

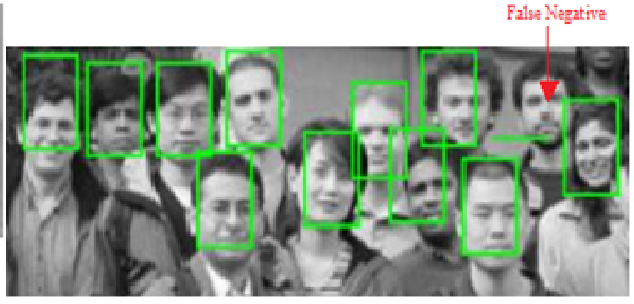

(d) Face detection with false negative

Figure. 11. Samples of tested Images and the results of detection

Based on the Experimental the proposed recognition system is compared with other well known benchmark face databases including the ORL, UMIST, Yale standard and Own database are shown in the Table. 2 
Signal \& Image Processing : An International Journal (SIPIJ) Vol.4, No.4, August 2013

Table 1. Statistical Data of Face Detection and Recognition

\begin{tabular}{|c|c|c|c|c|c|}
\hline $\begin{array}{c}\text { Test } \\
\text { Image } \\
\text { Number }\end{array}$ & $\begin{array}{c}\text { Number } \\
\text { of faces }\end{array}$ & $\begin{array}{c}\text { False } \\
\text { Positives }\end{array}$ & $\begin{array}{c}\text { False } \\
\text { Negatives }\end{array}$ & $\begin{array}{c}\text { Faces } \\
\text { detected }\end{array}$ & $\begin{array}{c}\text { Faces } \\
\text { recognized }\end{array}$ \\
\hline 1 & 2 & 0 & 0 & 2 & 2 \\
\hline 2 & 3 & 0 & 1 & 2 & 2 \\
\hline 3 & 4 & 0 & 0 & 4 & 4 \\
\hline 4 & 4 & 1 & 0 & 4 & 4 \\
\hline 5 & 5 & 0 & 1 & 4 & 4 \\
\hline 6 & 9 & 1 & 1 & 8 & 7 \\
\hline 7 & 6 & 0 & 0 & 6 & 6 \\
\hline 8 & 8 & 0 & 1 & 7 & 7 \\
\hline 9 & 10 & 1 & 0 & 10 & 9 \\
\hline 10 & 12 & 0 & 1 & 11 & 10 \\
\hline 11 & 23 & 2 & 1 & 22 & 21 \\
\hline 12 & 16 & 02 & 1 & 15 & 15 \\
\hline 13 & 60 & 02 & 02 & 58 & 57 \\
\hline 14 & 13 & 00 & 00 & 13 & 12 \\
\hline Total & $\mathbf{1 7 4}$ & $\mathbf{9}$ & $\mathbf{0 8}$ & $\mathbf{1 6 6}$ & $\mathbf{1 6 0}$ \\
\hline
\end{tabular}

Face Detection efficiency $=$ Number of faces - False Negatives

$$
\begin{gathered}
\text { Number of faces } \\
=(174-08) / 174=\mathbf{9 5 . 4} \%
\end{gathered}
$$

Face Recognition efficiency $=$ Number of faces recognized $=160 / 166=\mathbf{9 6 . 3 8} \%$ Number of faces detected

Table 2. Recognition rates for different standard Database

\begin{tabular}{|l|l|l|l|l|}
\hline Database & ORL & UMIST & Yale & $\begin{array}{l}\text { Own } \\
\text { Database }\end{array}$ \\
\hline Recognition rate & $100 \%$ & $95 \%$ & $95 \%$ & $100 \%$ \\
\hline
\end{tabular}

\section{Conclusions}

In this paper, to perform Gabor Wavelets neural network and Morphological Shared Weighted Neural Network approaches in a single framework of automatic face recognition system. The contribution in these approaches is that they extract wavelet coefficients from the original images followed feature extraction. In the next stage the Morphological Shared-weight Neural Network methods are applied on Feature extraction is performed on gray scale images using hit-miss transforms that are independent of gray-level shifts. Then the output is learned by interacting with the classification process. The system carried out a variety of experiments on standard datasets and also on our own dataset of image owing to different illumination conditions and cluttered background in non frontal images with a crowded scene with different conditions. The MSNN performs human faces in unconstrained environments, and multi-view recognition. 
Signal \& Image Processing : An International Journal (SIPIJ) Vol.4, No.4, August 2013

\section{REFERENCES}

[1] R. C. Gonzalez and R. E. Woods, Digital Image Processing Reading, MA: Addison-Wesley, 1992.

[2] Anil K. Jain, Arun Ross, and Salil Prabhakar, An Introduction to Biometric Recognition, IEEE transactions on circuits and systems for video technology, vol. 14, No. 1, January 2004, pp. 4-20

[3] W. Zhao, Rama Chellappa, A. Rosenfeld, and J. Phillips. Face recognition: A literature survey. ACM computing surveys (CSUR), V. 35, Issue 4, pp. 399458, 2003.

[4] P. Jonathon Phillips1, Patrick J. Flynn2, Todd Scruggs3, Kevin W. Bowyer, Overview of the Face Recognition Grand Challenge, IEEE computer society conference on computer vision and pattern recognition, 2005, vol. 1, pp. 947-954

[5] Cha Zhang and Zhengyou Zhang : A Survey of Recent Advances in Face Detection, Technical report is extracted from an early draft of the book Boosting-Based Face Detection and Adaptation.( 2010)

[6] Anissa Bouzalmat, naouar Belghini, Arsalane Zarghili and Jamal Kharroubi. : Face Detection and recognition using back progration neural Network and Fourier gabor filters, Signal \& Image Processing :An International Journal(SIPIJ), Vol.2, No.3, pp.15-21(2011)

[7] M. Mohammad Abadi, M. Rezapour, R. Jahani and Hadi Chahkandi Nejad : Face Detection with the help of Gabor Wavelets Characteristics and Neural Network Classifier, American Journal of Scientific Research, issue 36, pp. 67-76 (2011)

[8] Christian Tenllado, José Ignacio Gómez, Javier Setoain, Darío Mora, Manuel Prieto : Improving face recognition by combination of natural and Gabor faces",Elseiver journal of Pattern Recognition, pp. 1453-1460 (2010)

[9] Paul D. Gader,Mohamed A Khabou and Alexander Koldobsky : Morphological regularization neural networks, Elsevier Journal of the Pattern Recognition, Volume 33, Issue 6, Pages 935-944 (2000)

[10] Y. Won et. al., Morphological Shared-Weight Networks with Applications to Automatic Target Recognition, Electronics and Telecommunications Research Institute, Daejon, South Korea, (1995)

[11] Avinash Kaushal and J P S Raina, Face Detection using Neural Network \& Gabor Wavelet Transform , International Journal of Computer Sceince, volume 1, issue 1,2010, pp 58-62. 